Nathan WEINSTOCK, 1891-1907 : le mouvement sioniste découvre l'existence des Arabes de Palestine

Paris, Honoré Champion, 2016, 153 p.

\title{
Aline Schlaepfer
}

\section{(2) OpenEdition}

Journals

Édition électronique

URL : https://journals.openedition.org/assr/45272

DOI : 10.4000/assr.45272

ISSN : $1777-5825$

Éditeur

Éditions de l'EHESS

Édition imprimée

Date de publication : 1 décembre 2018

Pagination : 385-388

ISSN : 0335-5985

Référence électronique

Aline Schlaepfer, « Nathan wEInstock, 1891-1907 : le mouvement sioniste découvre l'existence des Arabes de Palestine », Archives de sciences sociales des religions [En ligne], 184 | octobre-décembre 2018, mis en ligne le 01 décembre 2018, consulté le 15 janvier 2022. URL : http:// journals.openedition.org/assr/45272 ; DOI : https://doi.org/10.4000/assr.45272

Ce document a été généré automatiquement le 15 janvier 2022.

(c) Archives de sciences sociales des religions 


\title{
Nathan WEINSTOCK, 1891-1907 : le mouvement sioniste découvre l'existence des Arabes de Palestine
}

Paris, Honoré Champion, 2016, 153 p.

\author{
Aline Schlaepfer
}

\section{RÉFÉRENCE}

Nathan WEINSTOCK, 1891-1907 : le mouvement sioniste découvre l'existence des Arabes de Palestine, Paris, Honoré Champion, 2016, 153 p.

1 Comment les sionistes du temps de la première aliya ont-ils traité de la question palestinienne? Ils l'ont entièrement occultée, assureront certains. "Un peuple sans terre pour une terre sans peuple. » D'autres répondront que si la rhétorique de la terre sans peuple a certes longtemps persisté dans les milieux sionistes, il n'en demeure pas moins qu'au tournant $\mathrm{du} \mathrm{xx}^{\mathrm{e}}$ siècle déjà, on publiait des essais visant à ouvrir un débat sur cette question. C'est le parti que prend Nathan Weinstock dans 1891-1907: le mouvement sioniste découvre l'existence des Arabes de Palestine. Il y propose une traduction, accompagnée d'une introduction et d'un commentaire de deux contributions importantes, mais néanmoins peu connues, à la pensée sioniste : «Vérité de la Terre d'Israël » de Ahad Ha'am paru en 1891 dans la revue HaMelitz à Saint-Pétersbourg et « Une question occultée » d'Yitzhaq Epstein, dans HaShiloah à Odessa en 1907.

2 Globalement, les textes de $\mathrm{Ha}$ 'am et d'Epstein reflètent les préoccupations caractéristiques des penseurs de la première aliya. Les Palestiniens ne constituent à leurs yeux pas encore de véritable problème politique, et le ton trahit une attitude foncièrement paternaliste. Toutefois, ces deux textes présentent une rupture fondamentale avec la pensée sioniste alors dominante, en proposant une véritable réflexion sur les possibilités de coexistence avec les agriculteurs palestiniens présents sur les territoires convoités par les premières vagues d'immigration sioniste, et sur les 
problèmes que devrait soulever l'appropriation de ces terres par les sionistes. Contrairement à nombre de leurs contemporains sionistes, auxquels Ha'am et Epstein reprochent d'ailleurs d'avoir "oublié » et «dissimulé » la question palestinienne, les deux auteurs identifient et dénoncent, amèrement et sans détour, une attitude qu'ils qualifient d'«arrogante " à l'égard des Palestiniens. Ils cherchent, chacun à leur manière, à anticiper la violence des réactions palestiniennes, qui devraient tantôt se trouver massivement privés de leurs terres. Il s'agit là d'une judicieuse sélection de la part de Nathan Weinstock, les deux textes se complétant de manière remarquable. Chez Ahad Ha'am, qui écrit au retour d'un voyage en Terre sainte, c'est moins la connaissance approfondie du terrain qui fait la force de son texte - n'étant lui-même pas résident sur place - que la critique qu'il adresse à ses contemporains sionistes. À l'inverse, Yitzhaq Epstein, installé depuis plus de vingt ans dans la région lorsqu'il publie sa "Question occultée », nous livre un tableau détaillé des conditions de vie et de travail des agriculteurs palestiniens.

3 Le texte d'Ahad Ha'am représente une source remarquable d'informations sur la complexité des discussions d'alors au sujet de la Palestine. Son essai révèle les fractures profondes, les dysfonctionnements majeurs au sein des sociétés sionistes nouvellement établies, l'importance des rivalités d'alors avec d'autres populations, comme la Société allemande des Templiers, de même que les effets négatifs de la dépendance financière des pionniers sionistes aux grandes philanthropies juives de l'étranger. Son point de vue sur les agriculteurs palestiniens sert, dans ce cadre, à alimenter son virulent réquisitoire contre les colons de la première aliya et apparait, en conséquence, truffé d'intéressantes contradictions. Si, comme le souligne Weinstock, Ahad Ha'am a régulièrement recours à la rhétorique classique de l'orientalisme, en dressant un portrait négatif et condescendant des populations arabes locales, paresseuses et prisonnières d'un mode de vie ancestral, les Palestiniens sont, en d'autres occurrences, les travailleurs agricoles formés, efficaces et expérimentés que le peuple d'Israël, qui est alors encore loin de l'autosubsistance, ne parviendra jamais à devenir : "Je ne suis parvenu à trouver, ne fût-ce qu'une seule personne, en mesure de vivre uniquement des produits de sa terre. [...] Les Arabes travaillent la terre et en retirent le nécessaire à leur subsistance, les Allemands travaillent la terre et en retirent le nécessaire à leur subsistance. Il n'y a que sur nous que "le Seigneur ait laissé éclater sa colère". Mais pourquoi? » (p. 86).

4 La connaissance approfondie du terrain, qui fait défaut dans le texte d'Ahad Ha'am, apparaît très clairement dans celui d'Yitzhaq Epstein. L'analyse de la complexité des conditions socio-économiques du fellah (le paysan palestinien), qui est au cœur de son étude, l'amène à la conclusion que « comme tout être humain, l'Arabe est très attaché à sa patrie» (p.98). Sa description détaillée du long processus d'acquisition du village druze de Metula au nord, entre 1897 et 1898, constitue certainement la partie la plus intéressante du texte. La peqidouth (administration) des fonctionnaires du baron Edmond de Rothschild n'étant parvenue à aucune forme de négociation avec la population avant leur départ forcé pour Istanbul suite à un soulèvement druze, celle-ci entra en contact avec les derniers habitants restés sur place, accompagnée d'« un officier de l'armée, suivi de ses soldats, venu arrêter ceux qui s'étaient soustraits au service militaire [...] et se tenant à prêts à emprisonner ceux qui refuseraient de signer les actes de vente»(p.103). Cette difficile négociation, affirme-t-il, exposa les communautés sionistes au ridicule, révéla leurs faiblesses, et alimenta plus encore la « haine profondément ancrée dans [les] cœurs ». Ainsi, tout au long de son argument, 
Epstein s'applique-t-il à déconstruire la rhétorique de la terre sans peuple : «L'heure est venue d'extirper des esprits l'idée discréditée, répandue parmi les sionistes, que l'on trouve en Eretz Israël des terres incultes par suite du défaut de main-d'œuvre et de l'indifférence de ses habitants. Il n'existe pas de champs inoccupés. C'est tout le contraire : chaque fellah s'efforce d'agrandir le terrain dont il dispose en y adjoignant la terre en friche qui est contiguë pour autant que cela ne requière pas un labeur excessif» (p. 97). La démarche d'Yitzhaq Epstein semble n'avoir pas été isolée. Ses propos sont en de nombreux points semblables à une série de dix-sept articles traitant de la condition des paysans parue dans la revue arabe Filastin entre 1911 et 1912, et dont le nom de plume de l'auteur, Abu Ibrahim, dissimule, en toute vraisemblance, le fruit d'une collaboration entre 'Isa al-'Isa, le fondateur de la revue, et l'agronome sioniste Menashe Meïrovitch (voir, Samuel Dolbee, Shay Hazkani, «"Impossible is not Ottoman", Menashe Meirovitch, 'Isa al-'Isa, and Imperial Citizenship in Palestine », International Journal of Middle East Studies, 47-2, 2015, p. 241-262).

Nathan Weinstock consacre une partie de son introduction aux idéologies nationales dominantes de la période en Palestine, à savoir le mouvement national juif sioniste et l'émergence de la conscience nationale palestinienne. Cette tentative de mise en regard des deux mouvements nationaux est louable car elle fait cruellement défaut à l'historiographie sur la région. Les acquis des historiens modernistes sur les nationalismes - qui voient dans l'émergence des idéologies nationales au XIX ${ }^{e}$ siècle, non pas le fruit de retrouvailles miraculeuses avec les glorieuses nations antiques tombées dans l'oubli au Moyen Âge, mais bien au contraire le fruit de créations, d'imaginations et $d$ 'inventions modernes d'identités collectives diverses (Benedict Anderson, L'imaginaire national: réflexions sur l'origine et l'essor du nationalisme, La Découverte, 1996 [1983] ; Eric Hobsbawm, Terence Ranger, éd., L'Invention de la tradition, Éditions Amsterdam, 2006 [1983]; Anne-Marie Thiesse, La création des identités nationales : Europe, $\mathrm{XVIII}{ }^{e}-\mathrm{Xx}{ }^{e}$ siècle, Éditions du Seuil, 2001) - ne semblent pas être remis en cause par l'auteur, qui analyse et décortique avec succès la fabrique de la nation juive à l'ère moderne. En parlant du Yishouv, l'auteur affirme qu'à partir de la fin du XIX siècle, « les Juifs de Palestine ont donné naissance à une nation nouvelle hébréophone » (p. 57). Pour ce qui est de la fabrique nationale palestinienne en revanche, l'auteur utilise la nature artificielle de l'identité palestinienne comme prétexte pour la décrédibiliser. Il rappelle ainsi à plusieurs reprises que la Palestine en tant qu'unité territoriale politique distincte était inexistante avant le $\mathrm{xx}^{\mathrm{e}}$ siècle (p. 13, 23, 116). Cet argument, qui refait régulièrement surface dans les espaces médiatiques, en particulier dans les moments de crise au Proche-Orient, vise souvent à discréditer les mobilisations palestiniennes courantes. En décembre 2011, le candidat républicain aux primaires présidentielles Newt Gringrich faisait débat lorsqu'il déclarait lors d'une interview: "Remember, there was no Palestine [...] We've had an invented Palestinian people.» Puis, lorsque la notion de Palestine commence à émerger dans les esprits et sur les cartes britanniques, ce n'est qu'à travers le prisme du rejet de la pensée sioniste ou de l'établissement d'un foyer juif en Palestine, affirme Weinstock, qui semble ignorer la pléthore de littérature sur l'émergence de l'idée de Palestine à travers les textes. La multiplicité des outils philosophiques, légaux et historiques, mobilisés pour penser, inventer et imaginer la collectivité et l'espace palestiniens à l'heure de la fabrique des nations a ainsi fait l'objet de nombreuses études (par exemple Rashid Khalidi, L'identité palestinienne : la construction d'une conscience nationale moderne, La Fabrique, 2003 [1997]). Dans son ouvrage intitulé Remembering and Imagining Palestine (Palgrave Macmillan, 
2008), Haim Gerber s'intéresse à l'émergence d'une identité palestinienne avant l'occupation britannique et démontre la récurrence du terme "Palestine", accompagné de la formule «notre pays", dans les sources arabes locales, bien avant l'arrivée des premières aliyot.

6 Le survol historique peu conventionnel que propose Weinstock doit par ailleurs faire face à une contradiction à plus large échelle. D'une part, l'auteur cherche à montrer la solidité des liens qui ont uni, à certains moments de l'histoire, les communautés juives et musulmanes dans les espaces arabo-musulmans en général, et au Levant en particulier, à travers la figure de Daher al-Zaydani au XVIII siècle par exemple. Il examine à ce titre une série de contextes dans lesquels des personnalités juives comme Haim Farhi occupèrent des positions privilégiées dans ces espaces socio-politiques à majorité musulmane. Pourtant, dans le chapitre suivant, Weinstock s'applique à démontrer « l'avilissement permanent » (p. 30) des juifs à l'islam, en raison du statut de dhimmi - terme regroupant les communautés principalement juives et chrétiennes des Empires musulmans, dont l'État doit protection en l'échange d'une lourde taxe. Comment expliquer alors ces multiples collaborations entre juifs et musulmans, si le contexte est caractérisé par un assujettissement quasi-atavique des juifs à la religion de l'Empire? Weinstock s'efforce, pour résoudre la contradiction, de présenter une série d'exceptions en précisant que " la mise en œuvre de ces règles a varié à l'extrême selon les époques, les lieux et la classe sociale à laquelle appartenaient les dhimmis, c'est-àdire les Infidèles astreints à ce régime discriminatoire " (p. 31). Très éloignés des préoccupations du pouvoir central, les agissements des communautés juives et chrétiennes échappaient en effet souvent au contrôle des administrations impériales. Leurs pratiques juridiques, socio-politiques et commerciales n'étaient par conséquent pas toujours liées à leur statut de dhimmi. Ceci ne signifie pas que leur condition était nécessairement meilleure ou moins bonne, mais simplement que la complexité de leur histoire ne saurait être réduite au seul statut de dhimmi. On pourrait, en d'autres termes, suggérer à Nathan Weinstock de se défaire du concept désuet et anachronique de « dhimmitude » qu'il emprunte à l'essayiste Bat Ye'or.

7 Enfin, Nathan Weinstock tend parfois à tomber dans le piège de ses sources lorsqu'il aborde la question des composantes de la population palestinienne. Il dépeint la société palestinienne à l'image de ce qu'en interprètent Ha'am et Epstein : divisée entre une écrasante proportion de paysans (fellahin) d'une part, soumise au bon vouloir d'une minorité de grands propriétaires terriens, d'autre part. Pourtant, le survol historique que propose Weinstock passe complètement sous silence l'existence de l'importante classe moyenne, composée d'hommes d'affaires, entrepreneurs, banquiers, assureurs et autres encore, dont l'influence sera déterminante lors des premières mobilisations palestiniennes. Depuis plusieurs années, les travaux de Salim Tamari notamment s'appliquent à dépasser cette schématisation des récits historiques traditionnels, entre l'aristocratie et la paysannerie palestiniennes ( $«$ Factionalism and Class Formation in Recent Palestinian History ", in Roger Owen, éd., Studies in the Economic and Social History of Palestine in the Nineteenth and Twentieth Centuries, 1982, p. 177-202 ; La montagne contre la mer. Essais sur la société et la culture palestiniennes, 2011 [2005]). Ceux que Sherene Seikali nomme Men of capital (2016) alimentèrent ainsi l'idée d'une nécessaire renaissance idéologique, progressiste, et économique de la Palestine, permirent à l'idéologie nationaliste de se diffuser à travers la presse, et financèrent in fine les révoltes palestiniennes. 
8 La publication d'une idée, aussi avant-gardiste et engagée qu'elle puisse être, ne suffit guère à assurer sa postérité, et Nathan Weinstock en est bien conscient (p. 132). Dans la partie conclusive de son livre - il est au passage regrettable de voir le cœur de sa réflexion relégué au rang de "quelques réflexions pour conclure »-, celui-ci nous livre une analyse fort intéressante de la réception de ces textes. Les propos parfois virulents d'Ahad Ha'am, notamment à l'encontre de Theodore Herzl, lui valurent toutes sortes de critiques, mais sa pensée fut malgré tout assimilée aux courants dominants, au point d'être aujourd'hui considérée comme fondatrice du mouvement sioniste dit culturel. À l'inverse, Weinstock nous apprend que l'accueil de la pensée d'Epstein fut loin d'être des plus chaleureux. En dehors de quelques réponses extrêmement hostiles (Y. Ben Tsvi, Klausner, Jabotinsky et Pukhachewsky), qui contribuèrent à crisper le débat plus qu'à l'enrichir, ses idées ne connurent pas de véritable postérité. Pourtant, Joseph Klausner, alors rédacteur en chef de la revue HaShiloah dans laquelle Epstein publie sa "Question occultée ", reconnaît en introduction l'utilité de ce texte, présenté sous forme de conférence au septième Congrès de Bâle deux ans plus tôt, pour la nécessaire amélioration du travail agricole en Palestine (Alan Dowty, "A Question That Outweighs All Others": Yitzhak Epstein and Zionist Recognition of the Arab Issue ", Israel Studies, 6-1, 2001, p. 34-54). 